\title{
Stannous fluoride toothpastes reduce the gingival index more than sodium fluoride toothpastes
}

\section{Are dentifrices containing stannous fluoride effective in reducing gingivitis?}

Paraskevas S and van der Weijden GA.

A review of the effects of stannous fluoride on gingivitis. J Clin

Periodontol 2006; 33:1-13

Data sources Medline and the Cochrane Central Register of Controlled Trials were used to identify sources of data.

Study selection Title and abstracts were screened independently. Randomised controlled clinical trials of $>6$ months duration that used gingivitis or plaque levels as outcome measures were selected.

Data extraction and synthesis The mean values and standard deviations were extracted. In studies where standard errors of the mean were reported, standard deviations were calculated by the authors based on the sample size. Weighted means of baseline and of end of trial were calculated with the software Cochrane Collaboration Review Manager (version 4.2; The Cochrane Collaboration, Oxford, England) using a randomeffects model. A meta-analysis for stannous fluoride (SnF) mouth rinse and dentifrice/ mouthrinse formulations was not performed because there were insufficient data.

Results Fifteen papers were included in the review. For SnF dentifrices, a statistically significant reduction in gingivitis was noted in comparison with control [weighted mean difference (WMD), 0.15 (gingival index) and 0.21 (gingivitis severity index); test for heterogeneity $\mathrm{P}<0.00001$, $I^{2}=91.1 \%$ and $P$ 50.03, $I^{2}=80.1 \%$, respectively]. There were inconsistent results for plaque reduction. On using the plaque index, no differences were found, whereas meta-analysis of the Turesky index gave a WMD of 0.31 (P 50.01; test for heterogeneity P 0.0001, $I^{2}=91.7 \%$ ).

Conclusions The use of $\mathrm{SnF}$ dentifrices results in greater gingivitis and plaque reduction compared with a conventional dentifrice. The precise magnitude of this effect was difficult to assess because of a high level of heterogeneity in study outcomes.
Address for correspondence: S Paraskevas, Department of Periodontology, Academic Centre for Dentistry Amsterdam ACTA, Louwesweg 1, 1066 EA Amsterdam, The Netherlands. E-mail: s.paraskevas@acta.nl

\section{Commentary}

As described in more detail below, the take-home message is that, yes, SnF toothpaste is more effective in reducing the gingival index than sodium fluoride $(\mathrm{NaF})$ toothpaste. Therefore, for patients with gingival inflammation, given a similar cost for the toothpastes, one could consider recommending SnF toothpaste.

This systematic review delineating these data, although confusingly written, convincingly demonstrates this point. Figure 1 and Table 1 below extrapolate from the review's data to provide another perspective. Figure 1 indicates that for all experiments identified in which $\mathrm{NaF}$ and $\mathrm{SnF}$ toothpaste were directly compared, ${ }^{1-6}$ the change in gingival index with use of SnF toothpaste was consistently more than the change in gingival index when a $\mathrm{NaF}$ toothpaste was used.

Similarly, Table 1 shows the number-needed-to-treat (the number of people needed to use SnF for one more person to have a change in gingival index) similarly indicates a consistent benefit of SnF compared with $\mathrm{NaF}$.

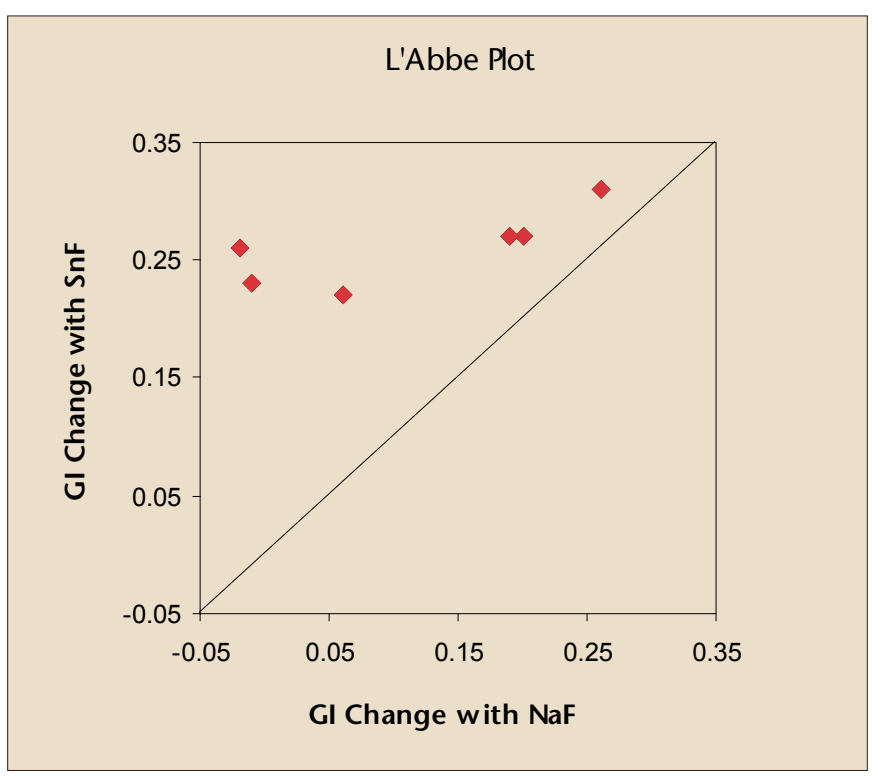

Figure 1. L'Abbe plot showing the change in Gingival Index (GI) in six studies

Another interesting observation is that approximately half of the identified studies were supported by a NaF toothpaste marketer, and the other half were supported by a SnF toothpaste marketer. Yet, given these conflicting perspectives, all the studies point in the same direction.

The lingering question, however, is how does the gingival index relate to the clinical situation? If the reports and the systematic 
Table 1. Change in gingival index (GI) in six studies and numbersneeded-to-treat (NNT)

\begin{tabular}{|c|c|c|c|c|}
\hline \multirow[b]{2}{*}{ Author } & \multicolumn{4}{|c|}{ Change in GI } \\
\hline & SnF & NaF & $\begin{array}{l}\text { Absolute risk } \\
\text { difference }\end{array}$ & NNT \\
\hline Perlich et al. ${ }^{1}$ & 0.27 & 0.2 & 0.07 & 14 \\
\hline Biswanger et al. $^{2}$ & 0.31 & 0.26 & 0.05 & 20 \\
\hline Biswanger et al. ${ }^{3}$ & 0.22 & 0.06 & 0.16 & 6 \\
\hline Williams et al. ${ }^{4}$ & 0.26 & -0.02 & 0.28 & 4 \\
\hline Mankodi et al. ${ }^{5}$ & 0.23 & -0.01 & 0.24 & 4 \\
\hline McClannahan et al. ${ }^{6}$ & 0.27 & 0.19 & 0.08 & 13 \\
\hline
\end{tabular}

review had converted the gingival index to a proportion of sites that bleed on probing (a typical clinical measure that is relatively easily obtained), this would be more clinically useful. Even better would be a measure of the teeth saved when using SnF or NaF toothpaste. Although this may seem ridiculous at first blush, this measure has in fact been successfully employed. ${ }^{7}$ Furthermore, from a patient's perspective, tooth loss is the most relevant outcome.

\section{Practice point}

SnF toothpaste is more effective in reducing the gingival index than $\mathrm{NaF}$ toothpaste. The magnitude of the effect is small, however, and the clinical effectiveness remains unclear.

\section{Richard Niederman}

Forsyth Institute, Boston, Massachusetts and Boston University Goldman School of Dental Medicine, Boston, Massachusetts, USA

1. Perlich MA, Bacca LA, Bollmer BW, et al. The clinical effect of a stabilized stannous fluoride dentifrice on plaque formation, gingivitis and gingival bleeding: a six-month study. J Clin Dent 1995; 6 (suppl.):S54-S58.

2. Beiswanger BB, Doyle PM, Jackson RD, et al. The clinical effect of dentifrices containing stabilized stannous fluoride on plaque formation and gingivitis - a six-month study with ad libitum brushing. J Clin Dent 1995; 6 (suppl.):S46-S53.

3. Beiswanger BB, McClanahan SF, Bartizek RD, Lanzalaco AC, Bacca LA, White DJ. The comparative efficacy of stabilized stannous fluoride dentifrice, peroxide/ baking soda dentifrice and essential oil mouthrinse for the prevention of gingivitis. J Clin Dent 1997; 8 (suppl.):S46-S53.

4. Williams $C$, McBride $S$, Bolden T, et al. Clinical efficacy of an optimized stannous fluoride dentifrice. Part 3. A 6-month plaque/gingivitis clinical study, southeast USA. Compend Contin Educ Dent 1997; 18 (suppl.):S16-S20.

5. Mankodi S, Petrone DM, Battista G, et al. Clinical efficacy of an optimized stannous fluoride dentifrice. Part 2. A 6-month plaque/ gingivitis clinical study, northeast USA. Compend Contin Educ Dent 1997; 18 (suppl.): S10-S15.

6. McClanahan SF, Beiswanger BB, Bartizek RD, Lanzalaco AC, Bacca L, White DI. A comparison of stabilized stannous fluoride dentifrice and triclosan/ copolymer dentifrice for efficacy in the reduction of gingivitis and gingival bleeding: six month clinical results. J Clin Dent 1997; 8 (suppl.):S39-S45.

7. Hujoel PP, Powell LV, Kiyak HA. The effects of simple interventions on tooth mortality: findings in one trial and implications for future studies. J Dent Res 1997; 76:867-874.

Evidence-Based Dentistry (2007) 8, 5-6. doi:10.1038/sj.ebd.6400507 